\title{
EDUCACIÓN DE JÓVENES Y ADULTOS: UNA EXPERIENCIA EDUCATIVA
}

$\mathrm{E}$

n este breve artículo, presentaré la experiencia del programa de Educación de Jóvenes y Adultos de la Universidad Mackenzie. La fundación de esta institución se dio en 1870 con la Escuela Americana, y fue reconocida como universidad en 1952.

Actualmente, la Universidad Presbiteriana Mackenzie (UPM) atiende a 25 mil alumnos, aproximadamente, y cuenta con 2 mil profesores, 27 cursos para la obtención de grados, 48 cursos de especialización, 8 cursos de maestría y un curso de doctorado en Administración. Asimismo, posee el campus São Paulo, 'Tamboré y Brasiliz, vinculado a la Facultad de Filosofia, Letras y Educación, y el Centro de Cultura y Extensión, responsable de los proyectos sociales orientados al área de Educación.

Los proyectos que se desarrollan son los siguientes: Programa de Alfabetización Solidaria Nacional, Grandes Centros Urbanos, Alfabetización Comunitaria (en Timor Leste), Educación de Jóvenes y Adultos, Universidad Solidaria, Tela de Saber, Escuela de la Familia, y Universidad Abierta del Tiempo UUtil. Todos ellos, con excepción del programa de Educación de Jóvenes y Adultos, y Universidad Abierta del Tiempo Útill, son colaboraciones realizadas 
con la Secretaría de Educación del Estado de São Paulo y del Ministerio de Educación.

Sobre el programa Educación de Jóvenes y Adultos (EJA), coordinado por Rosana Batista Monteiro, presentaremos, muy brevemente, una reseña histórica, sus principales objetivos, su propuesta educativa y su relevancia social, así como las actividades desarrolladas y los desafios para el futuro.

En 1993, el Departamento de Recursos Humanos de la UPM identificó la existencia de un gran nuimero de trabajadores que desarrollaban actividades simples, tales como limpieza, servicios generales, obras, entre otras, con poca o sin capacitación alguna. Ante esta situación, EJA fue creado con el objetivo de capacitar a algunos trabajadores de Mackenzie. Este programa no ofrece una certificación; es muy parecido, a pesar de ser más antiguo, a la propuesta de la Casa Edimar, relativa al cursillo de Escuela Técnica. Son ofrecidas clases basadas en el programa estipulado por el Ministerio de Educación que preparan a los alumnos para realizar los exámenes en órganos de la Secretaría de Educación, que certifica la conclusión de los estudios.

Considerando también que el problema de la educación debería ser tratado de forma adecuada y por profesionales capacitados, el programa, en 1997, pasó a ser coordinado por la Facultad de Filosofia, Letras y Educación, y se amplió para la comunidad.

El objetivo del programa es alfabetizar al ciudadano garantizando la continuidad de los estudios hasta la enseñanza media, de modo que responda a las exigencias de la realidad. En ese sentido, la relevancia social de este proyecto puede ser definida como un instrumento de inclusión social, dado que el público atendido está constituido por trabajadores de la Universidad, jóvenes y adultos de baja remuneración, gente de la calle, drogadictos y portadores de VIH. 
Resulta importante resaltar que el programa no quiere tencr un carácter asistencialista, sino que procura ser una propuesta de desarrollo de la humanidad de la persona: desea favorecer el desarrollo de su visión del mundo, descubrir la propia identidad sin negar la historia vital y la experiencia de cada uno.

Hoy en día, están matriculados 362 alumnos, en diez clases y en horatios de mañana y noche. Participan del programa 18 profesores, además de una coordinadora y el apoyo administrativo-educacional.

La característica principal del programa es la valorización del Yo. En los grupos del primer al cuarto ciclo es más visible la necesidad que tiene el joven, aunque principalmente el adulto, de ser observado por otro, de que su humanidad sea atendida por otro. En ese sentido, las experiencias son innumerables. Se percibe que el problema es menos conceptual y más de actitud, o sea, de percibirse, de poder decir «yo», a veces por primera vez. Entendemos que el hombre, joven o adulto, con quien nos reunimos diariamente tiene la necesidad de pertenecer. Cuando él pertenece a «algo» o a kalguien», a una institución o a una historia, su perspectiva cambia. Ese pertenecer les permite hacer una relectura y retomar su propia historia. En ese aspecto, un ejemplo muy bello es el relato de la coordinadora de un centro de acogida de portadores de VIH, para muchos de los cuales eta muy importante ir a Mackenzie, pertenecer a la institución, formar parte de un grupo, ser acogidos. Ella dice que esa relación provoca que los portadores de VIH enfrenten su dolencia con más coraje y realicen el tratamiento de manera correcta. Cuando ellos tienen baja estima, generalmente, no tienen voluntad de viviry no toman la medicación adecuada, mientras que aquellos que vienen a las aulas de EJA muestran una disposición nueva, una mirada diferente, 
Partiendo de la realidad concreta que viven nuestros alumnos, buscamos cl desartollo integral de la persona; por eso, la propuesta educativa valoriza también la cultura en su diversidad y busca rescatar las tradiciones populares que forman parte de la cotidianeidad de quienes participan del programa.

Además de las aulas regulares de Enseñanza Fundamental I y II, y de Enseñanza Media, el programa promueve la Semana Cultural EJA, realizada una vez por semestre, ocasión en que los alumnos presentan sus trabajos y participan en diversas actividades: teatro, danza y lectura de poemas. En este caso, la propuesta es que los propios alumnos se organicen para realizar las presentaciones, y los profesores comprometidos se sirven de la creatividad y de los recursos de audio, video y fotografia para valorar el producto cultural de estos.

Existe también el coro de EJA y de la UATU (Universidad Abierta del Tiempo Útil), que está compuesta en su mayoria por gente de la calle. La importancia de un gesto como ese en la vida de un habitante de la calle significa no solo una valoración en el aula por medio de la relación profesotalumno, sino ser valotado por una platea, que asiste y aplaude. La presentación del coro aconteció hace pocos dias y era impresionante ver cómo lo bello, la belleza, es la expresión de una verdad, Los ojos de sus integrantes eran otros: brillaban, sonreian. Para muchos, fue la primera vez que eran protagonistas de un acontecimiento. Ver la expresión del rostro, la postura humana de cada uno, el ir cambiando en el tiempo fue la evidencia de un Yo nuevo, de un Yo presente en la sociedad.

Los eventos culturales se complementan con visitas a teatros, museos, cines y cuudades históricas, buscando siempre partir de su realidad y favoreciendo el descubrimiento de cosas nuevas. Generalmente, las actividades son 
programadas por los profesores de las disciplinas que buscan relacionar el contenido de la propuesta. Los paseos son financiados por la Universidad o por convenio con la Secretaría de Educación o la Prefectura de São Paulo, Los alumnos de. EjA están también integrados a la comunidad académica, es decir, ellos tienen acceso a la biblioteca y a los laboratorios de informática y química, al igual que todos los alumnos de la Universidad. Asimismo, participan de la Caminata Solidaria, actividad realizada al empezar cada semestre para recibir a los cachimbos.

Esa integración se da también en el Curso de Pedagogia. Existe un convenio que posibilita a los alumnos, ya que tienen acceso a los servicios antes señalados, realizar cursos de inglés, informática, salud y nutrición, los cuales son preparados por las alumnas de Pedagogía mediante proyectos relacionados con las disciplinas de pregrado, como 'Tecnología Educacional, Biología de la Educación, Metodología de la Enseñanza de la Lengua Portuguesa, entre otras. Este vinculo es de gran relevancia para el pregrado, además de proporcionar a los alumnos de EjA acceso a recursos que posiblemente jamás hubieran podido conocer en otras circunstancias. Las clases, siempre llenas de alumnos, son realizadas los sábados y la participación es libre.

EJA tiene como perfil a la persona preocupada por aprender. Nuestros alumnos se dan cuenta de que cuanto más se van alejando de los primeros ciclos, cuando ya consiguen dar los primeros pasos por propia cuenta, pasan a exigir mayor contenido. Como ya se mencionó, prima una educación de lo humano al rescate del Yo, de la identidad, de la visión y percepción de la realidad, para que con esos elementos puedan dar pasos rumbo al desarrollo académico. Deseamos que la persona pueda partir de la realidad, que tenga elementos para identificar su presencia en la sociedad sin negar la condición 
en la que viven: reconocer que la diferencia es el Yo y la propia visión frente a la realidad.

En el caso de los habitantes de la calle es claro que objetivamente su realidad no va a cambiar, es decir, ellos continuarán viviendo en las calles; pero es su Yo el que cambia: dejan de ser violentos o excluidos y pasan a tener un Ingar. Muchos no consiguen emplea, pero, por ejemplo, pasan a recolectar latas en las calles y a formar parte de una cooperativa de reciclaje de desperdicios; otros van a constituir una familia. Es una mirada verdadera y positiva ante la realidad.

Hablando con la coordinadora del programa sobre las perspectivas y desafios que el EJA tiene para el futuro, podemos identificar que es necesaria una inversión de los objetos (inversión formal, porque, en la práctica, el enfoque es exactamente en lo humano), pues comprendemos que es mediante la valoración de la educación del ser humano que la conclusión de los estudios se vuelve una consecuencia.

Otra propuesta es la de un convenio con la UATU para una educación permanente, ello porque una vez que los alumnos no pasan las pruebas (ellos tienen el deseo de aprender y no se preocupan de la prueba formal) retornan cada semestre al mismo ciclo, lo que impide que otras personas puedan participar del programa. A partir de 2004, habrá un tiempo límite para la permanencia en cada ciclo, así como la propuesta de cursos vinculados a la UATU, hacia donde estas personas serán orientadas.

Considerando el problema de LDB 9394/96 (donde se reduce la edad, de 18 a 15 años, para la conclusión de los estudios en la escuela regular), los programas de EJA (por tanto, el nuestro también) pasaron a recibir alumnos con edades entre 16 y 75 años. Esa gran distancia de edad produce una infinidad de conflictos, ya que el joven aprende deprisa, mientras que al adulto le lleva 
un tiempo mayor desarrollar lo aprendido. En este caso, lo ideal seria la formación de grupos de jóvenes y de adultos por separados, atendiendo la necesidad de cada franja de edades. Sin embargo, existe un gran inconveniente, en la Universidad no hay espacio físico para la cesión de nuevas aulas; por ella, estamos buscando nuevos convenios con otras instituciones preocupadas por los jóvenes, como, por ejemplo, Acción Educativa.

Cabe señalar, finalmente, la necesidad de un profesor que no tenga un perfil asistencialista, sino que sea capaz de percibir la necesidad específica del alumno, corriendo el riesgo de infantilizar. Queda claro que algunas técnicas, pot ejemplo, de alfabetización, son las mismas, pero se debe enfocar hacia el significada. Para un niño, un ladrillo es un ladrillo; para un adulto, un ladrillo significa construcción, trabajo; en otros términos, es preciso enfocarse hacia la experiencia de aquel adulto.

Reconocemos que el programa es limitado en el sentido de que la certificación de los estudios no garantiza el empleo para este joven o adulto. Es necesario - y ese es un desafio- ejecutar acciones sociales que acojan a aquellas personas que estén por concluir los estudios y poder capacitarlos para el mercado de trabajo.

Con tales perspectivas y expectativas, y mediante lo que está a su alcance, EJA busca atender a una pequeña parte de la población; con ello, intenta velar por las necesidades reales de los jóvenes y adultos que participan de este programa. 